\title{
The pneumatisation of anterior clinoid process is not associated with any predictors that might be recognised preoperatively
}

\author{
T. Szmudaํ, P. Sloniewski ${ }^{1}$, A. Baczalska², M. Cabala², B. Czapski², A. Gorczynski², \\ N. Kreja², M. Kindrachuk ${ }^{2}$ \\ ${ }^{1}$ Neurosurgery Department, Medical University of Gdansk, Poland \\ 2Students' Scientific Association, Neurosurgery Department, Medical University of Gdansk, Poland
}

[Received 21 November 2012; Accepted 12 January 2013]

The anterior clinoid process (ACP) is usually removed during surgeries of proximal internal carotid artery (ICA) aneurysms. However, some ACPs present with air cells originating from the sphenoid orland ethmoid sinus. In surgeries containing a clinoidectomy of a pneumatised process, up to $40 \%$ of patients experience cerebrospinal fluid (CSF) rhinorrhoea. The aim of this study was to explore the potential predictors of pneumatisation of the ACP, as well as to compare the occurrence of CSF rhinorrhoea between total and partial anterior clinoidectomies. This study comprised 2 different groups, with 2 different analyses. Firstly, the pneumatisation of the ACP was evaluated in 496 ACPs and was based on 248 computer tomography exams (CT). The $\chi^{2}$ test and ROC curve comparisons were utilised in conjunction, to explore possible predictors of air cell accumulation in the ACP. The overall pneumatisation rate was 9.7\%, unilateral and bilateral aerial ACP was found in $4.4 \%$ and $2.6 \%$ of all patients respectively, while at least one pneumatised ACP was found in $14.1 \%$ of examined patients. The route of pneumatisation was established in $87.5 \%$ of cases. The side of the ACP, gender, and patient age were not significantly associated with both pneumatisation of ACP or route of pneumatisation. Secondly, a clinical group of 23 patients after operative securing of an ICA aneurysm were retrospectively assessed with regards to the extent of anterior clinoidectomy and the occurrence of CSF rhinorrhoea. A total of 23 ACPs were removed, 17 ACPs were totally resected, and 6 underwent partial resection. CSF rhinorrhoea was not noted in any patients, thus the comparison between clinical groups was not valid. Moreover, we described a novel method of partial removal of the lateral aspect of ACP, which was applied in 6 patients treated for an ICA - ophthalmic artery junction aneurysm. (Folia Morphol 2013; 72, 2: 100-106)

Key words: skull, middle cranial fossa, anterior clinoid process, internal carotid artery, sphenoid sinus, ethmoid sinus, anterior clinoidectomy, brain aneurysm, neurosurgical technique

Address for correspondence: T. Szmuda, MD, PhD, Neurosurgery Department, Medical University of Gdansk, ul. Dębinki 7, 80-952 Gdańsk, Poland, e-mail: tszmuda@gumed.edu.pl 


\section{INTRODUCTION}

The anterior clinoid process (ACP) is an intracranial, medial extension of the lesser sphenoid wing and represents a roof to the anterior segment of the cavernous sinus [16]. The process projects posteriorly and medially in the middle cranial fossa and constitutes a boundary of the caroticoclinoid foramen if the ACP is merged with the top of the medial clinoid process [10]. The ACP is closely related to many vital neurovascular structures, including the internal carotid artery (ICA) adjacent to the medial and posterior aspect of the ACP $[8,22]$. The relationship between these structures can be pathologically affected by growing with expansion and lateral projection of an ophthalmic artery aneurysm or paraclinoid ICA aneurysm [18]. In such cases the ICA is mildly repositioned medially towards the optic strut, and consequently the ACP may completely or partially block the neck of the aneurysm. In patients afflicted with any of the aforementioned aneurysms, intraoperative extra- or intradural removal of the ACP (anterior clinoidectomy) is an essential manoeuvre to expose the aneurysm neck in order to ensure safe clip placement. Likewise, treatment of some supraclinoid aneurysms, basilar artery aneurysms, giant pituitary adenomas, and cavernous sinus lesions also require an anterior clinoidectomy, which is an important step in an extended neurosurgical approach [1, 3, 12, 14, 23, 24]. In cases of suprasellar meningiomas, recurrent growth is prevented by removal of the bony tumour origin, including the ACP; therefore, an anterior clinoidectomy is also used in the treatment of such patients. Furthermore, the decompression of the optic canal as well as enhancement of neurosurgical exposures to many surrounding structures in the approach is additionally facilitated by the removal the ACP [7, 14]. Regardless of the multiple techniques in preforming an anterior clinoidectomy $[6,8,25]$, cerebrospinal fluid (CSF) rhinorrhoea is a common complication in up to $40 \%$ of patients undergoing this procedure [22]. This is due to the fact that the process is pneumatised by the sphenoid, ethmoid, or both sinal origins [14]. Apart from rhinorrhoea, anterior clinoidectomies increase the rate of oculomotor nerve paresis, injuries of ICA, ophthalmic artery and cavernous sinus, or intraoperative aneurysm rupture [2, 6, 18, 22]. However, in recently published cadaveric and radiographic studies pneumatised ACPs pertained to a minority of patients and ranged from $4 \%$ to $29 \%$ of investigated cases $[1,4,5,14,20,21]$.
Nowadays, Gamma Knife therapy for meningiomas that invade the cavernous sinus and endovascular coiling of paraclinoid aneurysms are alternative options in standard neurosurgical methods [14]. Thus, attention to detail with regards to complication avoidance is up to date. Radiographic evaluation of air cell infiltrate into the ACP in all cases, including any of the previously mentioned indications, is recommended if bony destruction in the middle cranial fossa is considered preoperatively $[10,14,22]$. However, in emergent situations, such as patients with a ruptured aneurysm, the preoperative assessment of pneumatisation is occasionally abandoned. The investigation of potential simple factors, like gender and side, allowing for predictions in accordance with pneumatised or osseous ACPs is plausible for neurosurgeons. Our study discusses the above issue and presents information pertaining to the pneumatisation rate of the ACP in white Caucasian populations. We demonstrated restricted lateral side anterior clinoidectomies in laterally projecting paraclinoid and ophthalmic artery aneurysms, while limiting postoperative rhinorrhoea. The comparison between total and partial removal of ACP in terms of CSF rhinorrhoea occurrence is presented as well. No previous studies regarding these issues have been published to date.

\section{MATERIALS AND METHODS}

The study consisted of 2 investigative steps, in which 2 different groups were included. Firstly, pneumatisation of ACP was evaluated by means of computer tomographies (CTs) in patients without skull base pathology. Secondly, the risk of CSF rhinorrhoea after total or partial ACP removal was assessed in a clinical group of patients treated due to laterally projecting ophthalmic/paraclinoid ICA aneurysms. In these 2 steps 2 different populations were investigated in a university hospital in Europe, where the study originates.

In the first part of the study, multidetector-row and high-resolution head CTs of 248 patients were studied. CTs were acquired using a GE LightSpeed VCT (from 2010 to 2011) and a Siemens Somatom (in 2012). Exams were obtained and recorded in DICOM format and slices were $1.25 \mathrm{~mm}$ in thickness. A group of $248 \mathrm{CTs}$, collected between January 2010 and August 2012, were selected for this study. Exams included in the analysis were performed in relation to neurovascular disease, brain tumours, trauma, or screening. All exams were verified retrospectively in 2012 by a neurosurgeon (senior author). The revision resulted in the exclusion of 8 exams, in which presenting pathologies involved the skull base. 


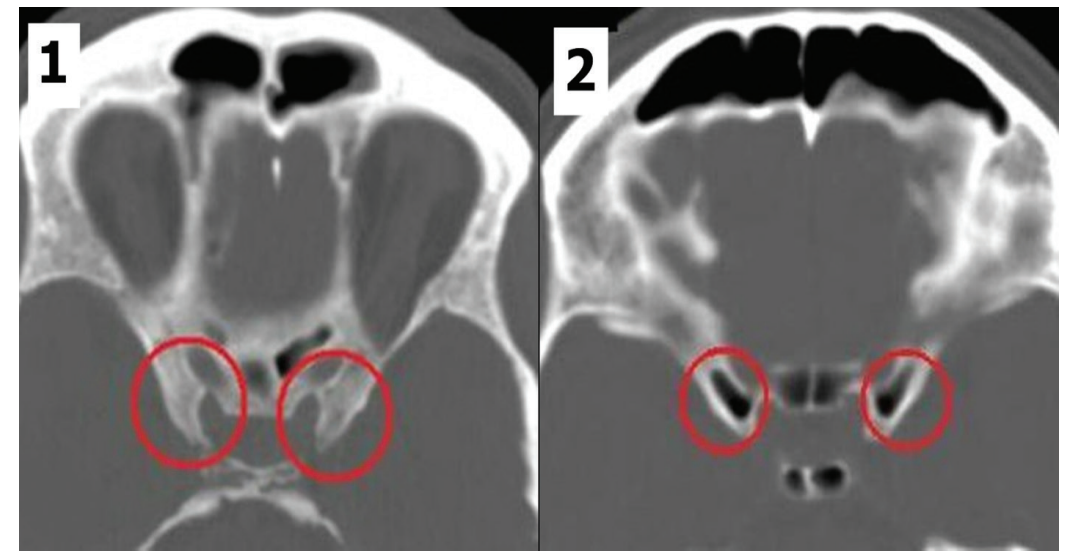

Figure 1. Pneumatised and osseous anterior clinoid process (ACPs; marked by red circles) visualised by computer tomography (CT) imaging; 1 - both ACPs are osseous; 2 - bilateral pneumatisation of ACP.
These pathologies were suspected to alter the natural anatomy of ACP. The pneumatisation of the process, as well as its connection with the sphenoid or ethmoid sinus, were verified by means of axial, sagittal, coronal, and multiplanar reconstructions using digital image processing software (Osirix Software, version 4.1.2 for Mac OS, Pixmeo SARL Co, Switzerland). The division of ACPs into pneumatised or osseous and sinal connection tracking was performed with 'bone' (window/level = 300/2000) and 'sinus' (window/level $=400 / 4000$ ) imaging settings. The age, gender, and side of the skull were investigated for potential association with the pneumatisation of the $A C P$, and its sinal connections.

Selection of statistical testing was based on the distribution of continuous variables verified by the Kolmogorov-Smirnov test. Student's t-test and its non-parametric equivalent Mann-Whitney $U$ test were applied for evaluative comparison. $\chi^{2}$ with Yates correction for continuity was used to compare categorical variables. Descriptive statistics, including mean and median value, standard deviation (SD), and range were included. Software Statistica v.10.0 (StatSoft, Inc.) was used for statistical analysis, with the significance level $<0.05$ in calculations. Receiver operating characteristic (ROC) curves and area under ROC curves (AUC) were subsequently calculated to complement the prognostic value of given factors in pneumatisation of ACPs, as well as in sinal connection with the ACP.

The study group comprised 102 (41.1\%) females and 146 (58.9\%) males. Mean and median ages were 58.4 and 61.0 years, respectively, while ages ranged from 18 to 101 years (SD \pm 19.5 ). The group was homogeneous as no significant correlation between gender and age was observed ( $p=0.26)$.

The second part of the study consisted of a clinical group of 23 patients, who underwent neurosurgically secured ophthalmic, paraclinoid, or supraclinoid ICA aneurysms between the years 2007 and 2012. Pterional craniotomy followed by extradural medial superior orbital fissure opening was conducted in all cases. Standard total anterior clinoidectomy was performed in 17 cases harbouring ICA aneurysms, whereas 6 patients presented with laterally projecting ophthalmic aneurysms, in which case partial removal of the lateral aspect of the ACP was performed. A retrospective analysis of surgical video recordings with regards to the extent of the anterior clinoidectomy, as well as a retrospective review of medical documentation for CSF rhinorrhoea, were performed.

\section{RESULTS}

\section{Pneumatisation of ACP}

There were 248 patients examined in the study, thus, totalling 496 ACPs. Air cells were encountered in 48 of the processes, resulting in overall pneumatisation of $9.7 \%$ (48 of 496). Pneumatisation of at least one ACP was found in $14.1 \%$ of patients (35 of 248). Of that group, $62.9 \%$ (22 of 35) had one aerial ACP and $37.1 \%$ (13 of 35 ) bilaterally. If all examined patients were taken into consideration, unilateral and bilateral pneumatised ACPs were observed in 4.4\% (22 of 248) and $2.6 \%$ (13 of 248) of CT exams, respectively (Fig. 1).

The side of ACP did not present as a significant predictor of pneumatisation ( $p=0.17$ ), although air cell presence in the process was observed more often on the left $(11.7 \% ; 29$ of 248$)$ than on the right side $(7.7 \% ; 19$ of 248$)$. There was a $(p=0.49)$ greater prevalence in process pneumatisation in males $(10.6 \%$; 31 of 292$)$ than in females $(8.3 \%$; 17 of 204). All the abovementioned factors failed in terms of predictive characteristics for pneumatisation of the $A C P$, as none of the AUC values exceeded 0.6 (side of ACP: $A U C=0.56,95 \% \mathrm{Cl} 0.51-0.60$; gender: $\mathrm{AUC}=0.53$, 


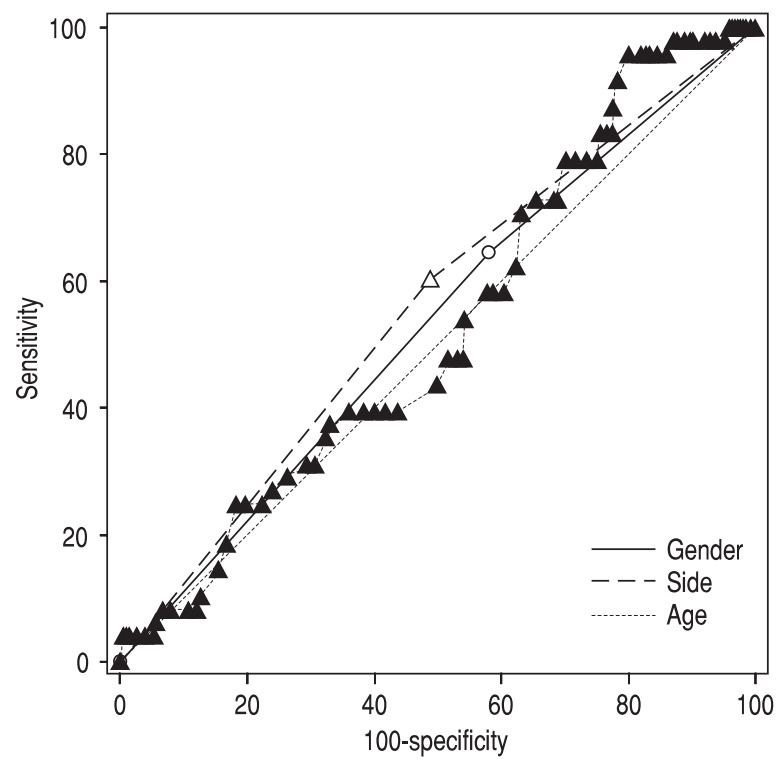

Figure 2. Predictions for pneumatisation of the anterior clinoid process (ACP). Receiver operating characteristic curves for gender, side of ACP, and age. Values of $x$ and $y$ axes are shown in percentages.

95\% Cl 0.49-0.58). The pneumatised or osseous ACP was found regardless of age $(p=0.33)$, although the mean age in the group with pneumatised ACP $(61.0 \pm 16.7)$ was higher than in the osseous process group (58.1 \pm 19.7$)$. Areas under the ROC curves were examined for the following subsequent variables: side of the process, gender, and age (Fig. 2).

A sinal connection of the ACP was noted in the majority of exams. However, there were $12.5 \%$ (6 of 48) of pneumatised processes, in which direct route of pneumatisation was not established. The origin of the air cells in ACP mostly referred to sphenoid (78.6\%; 33 of 42 ) and ethmoid sinuses exclusively (11.9\%; 5 of 42$)$, whereas in 4 cases $(9.5 \%$; 4 of 42$)$ both sinuses were involved.

Side of ACP $(p=0.43)$, gender $(p=0.73)$, and age $(p=0.63)$ did not influence the connection of the process to any sinus, sphenoid or ethmoid (all $p>0.05)$. ROC curves of variables such as side of the $A C P$, gender, and age were created. Side of ACP and gender failed in sinal connection prediction as all AUCs were below 0.6 (side of ACP: AUC $=0.54,95 \% \mathrm{Cl}$ 0.49-0.58; gender: $A \cup C=0.53,95 \% \mathrm{Cl} 0.49-0.58$ ) (Fig. 3).

\section{Total and partial anterior clinoidectomy}

Total anterior clinoidectomy was performed using the traditional operative method. The ACP was drilled

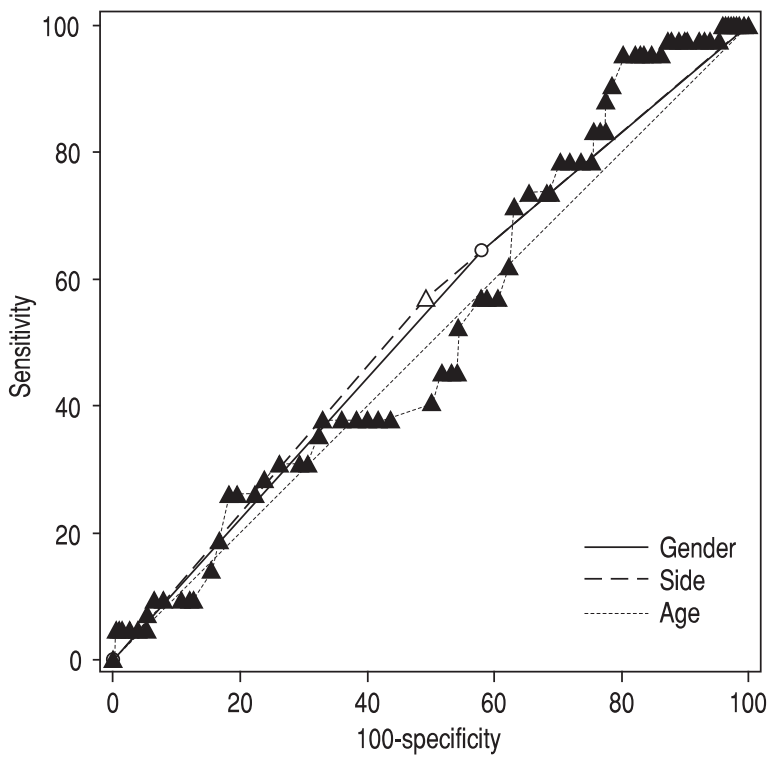

Figure 3. Prediction of connection between anterior clinoid process (ACP) and sinus occurrence. Receiver operating characteristic curves for gender, side of ACP, and age. Values of $x$ and $y$ axes are shown in percentages.

down from the top and followed by eggshell drilling of the process. Then, the base of the remaining medial part of the ACP was broken and separated from the optic strut. The surrounding attachments to the arachnoid dura were liberated, and unroofing of the optic canal was performed in the presence of a large or giant aneurysm (Fig. 4).

Partial anterior clinoidectomy was our proposal, to minimise damage to the skull base as well as to prevent nasal rhinorrhoea. An unobstructed neck of a laterally projecting ophthalmic aneurysm is essential for safe clipping, even if the removal is restricted to the lateral side of the process. Prior to the clip placement, opened air cells in the process were filled with a piece of muscle, haemostatic and/ /or fibrin glue, following a partial or total anterior clinoidectomy. Sealing of the ACP was performed to ensure a barrier for the prevention of CSF leakage, but this decreased access to the aneurysm in one case from our series (Fig. 5).

Cerebrospinal fluid rhinorrhoea complications were not noted in any patients who underwent a total or partial anterior clinoidectomy throughout the study period. Statistical assessment of the above negative clinical endpoint was not valid. In other words, no occurrences of postoperative nasal rhinorrhoea prevented comparison between the series of total and partial process osteotomy. 


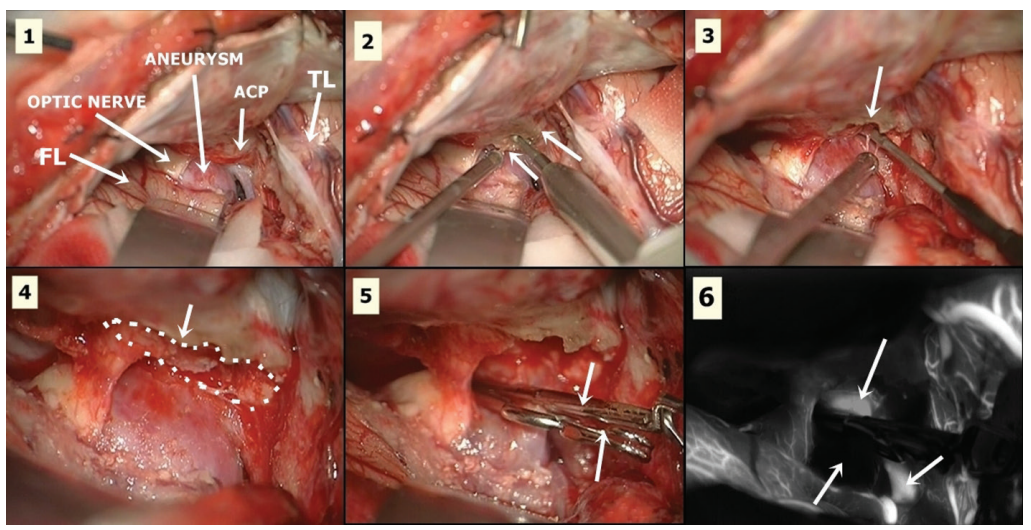

Figure 4. The steps in total removal of the anterior clinoid process (ACP) in an illustrative case portraying an unruptured, medially projecting, ophthalmic internal carotid artery (ICA) aneurysm (right side); 1 - ACP covered the entire neck of the aneurysm and partially the ICA (its proximal portion to the neck). ACP prevented securing the safe aneurysm. Optic nerve was flattened and stretched by the dome of the ICA aneurysm; 2 high-speed drill boring through pneumatised ACP, under operator's control (2 arrows). If pneumatisation of the process is unknown preoperatively, an unintended fall in of the drill may cause neural or vascular damage, 3 - following entire ACP resection, the aneurysm neck dissection was then available (arrow); 4 - access to the neck of the aneurysm was facilitated by total ACP removal. Further unroofing of the optic canal was to preserve the function of the optic nerve by its mobilisation. The extent of the osteotomy is marked by dashed white line and arrow; $5-$ two clips (arrows) were placed on the neck of the aneurysm, which had been previously revealed by total ACP removal; 6 — post-clipping blood flow in the vessels is confirmed by means of near-infrared intraoperative indocyanine green spectroscopy $(800 \mathrm{~nm})$. Two upper arrows show normal blood flow (white) in the proximal and distal portion of the ICA to the clipped neck of the aneurysm. Third, lower arrow indicates collapsed dome of the aneurysm without any blood flow (black); $\mathrm{FL}$ — frontal lobe; $\mathrm{TL}$ — temporal lobe.

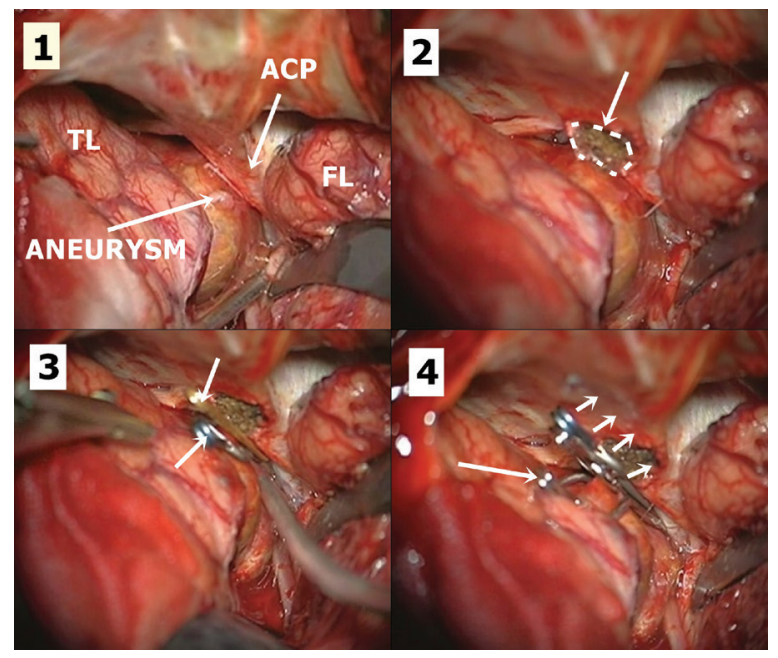

Figure 5. Steps of partial removal of the anterior clinoid process (ACP) in the approach to the neck of a laterally projecting ophthalmic internal carotid artery aneurysm (left side); 1 - lateral aspect of ACP covering the proximal neck of the aneurysm; 2 - the neck of the aneurysm revealed after partial removal of the osseous process. Dashed white line and arrow indicate the extent of the resection of the process; 3 - temporary parent vessel occlusion with a gold clip (upper arrow) allowed safe permanent silver clip placement (lower arrow); 4 - temporary gold clip was removed afterwards, while the addition of a second permanent clip (left arrow) facilitated the repositioning of the initial clip ( 4 arrows on the right side). The clip position resulted in total aneurysm occlusion and was accessible if followed by partial anterior clinoidectomy. In the presented case total ACP removal would be dispensable in our opinion; $\mathrm{FL}-$ frontal lobe; $\mathrm{TL}$ - temporal lobe.

\section{DISCUSSION}

The presented study consisted of two investigated series: radiological and clinical. The first step was to examine the radiological series for predictors of pneumatisation of the ACP as well as its sinal connections. The occurrence of aerial ACP significantly increases the incidence CSF rhinorrhoea $[17,18,25]$. The following investigation was to compare incidences of CSF rhinorrhoea between complete and incomplete anterior clinoidectomies in clinical groupings. These investigations of separate series concern the same issue, namely, finding predictors of CSF rhinorrhoea. The results are addressed either to the neurosurgical or anthropologic societies.

The knowledge of ACP anatomy and its relation to vital neurovascular structures is exceptionally important for skull base neurosurgeons. The ACP is a bony structure covered by dense cortical bone. The anterior/ superior (roof of the optic canal) and posterior/inferior (optic strut) roots of the lesser sphenoid wing form the bases of the process $[1,9,11]$. The ICA is adjacent to the medial inferior part of the ACP. Surgery involving lesions located close to the ACP or involving any part of the ACP necessitates an anterior clinoidectomy. This extension of the standard pterional approach has been the gold standard for years when approaching these pathologies $[2,6$, $17,18,26]$. While drilling, the surgeon must pay special attention to the ICA and the aneurysm wall. Furthermore, 
the preoperative evaluation of ACP facilitates safer high-speed drilling and is recommended by practitioners $[1,10]$. Intraoperative neural or vascular damage occurs more often in pneumatised ACP than in osseous examples. This is because the rotating drill may bore into the empty process with relatively high force and harm the surrounding structures, including the brain cortex, cranial nerves, ICA, orbital content, or cavernous sinus [2, 22]. Preoperative evaluation of the ACP is omitted in several emergent cases of ruptured ICA aneurysms encountered in normal practice. Therefore, the analysis of the bone window in CT imaging should not be discarded. Finding potential and consequently simple factors related to pneumatised ACP occurrence appears plausible in neurosurgical practice. Surprisingly, such investigations have not yet been presented in either anatomical or neurosurgical literature.

\section{Pneumatisation of ACP}

The majority of ACPs are osseous. The incidence of pneumatised processes has been reported as ranging from $4 \%$ to $29.3 \%$ in several studies $[1,4,5,14,20,21]$. Thus, up to one in 3 resected ACPs should be assumed to have air cells accumulated within. The incidence of pneumatised ACPs in our series was in accordance with cited reports. In our series, pneumatisation affected $9.7 \%$ of all processes, whereas at least one process was pneumatised in $14.1 \%$ of patients. The authors of a recent study [1] made a step forward in their analysis of the pneumatisation of ACP and created a 4-type classification of pneumatisation degree. According to their results, less than $50 \%$ pneumatisation degree was observed in $69.3 \%$ of cases, while totally pneumatised processes occurred in $17.7 \%$ of all affected ACPs. The authors attached importance to the clinical meaning of the proposed classification and explained that the drilling of empty processes is safer. We had the opposite opinion. Moreover, it seems to us that stratifying the ACP with regards to degree of pneumatisation does not meet the operating neurosurgeon's requirements. The extent of partial resections described in our study exceeds half of the ACPs width. However, air cells become open regardless of the extent of osteotomy. Creating classifications pertaining to the degree of pneumatisation is clearly invalid.

All of the investigated factors, including the side of the ACP and gender, failed in the prediction of pneumatised ACP occurrence. If any predictors exist, they remain unexplored. Other authors did not find any influence of gender and side of ACP on the increased incidence of pneumatised processes either $[1,14]$. However, our study did confirm the well-known principle that scrupulous CT imaging assessment should precede ACP removal in every case $[1,10]$. Patient age has not yet been investigated and compared between pneumatised and osseous ACPs. Our study revealed that process pneumatisation is not age-related. Two patterns of pneumatisation of the ACP are described: originating from the sphenoid sinus via the optic strut, and from the sphenoid sinus by the base of the ACP (anterior recess). Researchers found sphenoid sinus connection in $38.0 \%$ to $74.5 \%$ of processes and sphenoid sinus origin in $14.5 \%$ to $22.6 \%$ of aerial ACPs, while involvement of both sinuses in an individual are reported in $10.9 \%$ to $17.7 \%$ of cases $[1,14]$. The route of pneumatisation in our series was similar to the above reports; the aerial connection to sphenoid, ethmoid, and both sinuses was observed in $78.6 \%, 11.9 \%$, and $9.5 \%$ of cases, respectively. There were no differences in the route of pneumatisation between any genders or any sides of ACP as well as various patients' age. The association between all these factors and sinal origin have not been explored before although our study confirmed that the presence of aerated channels within the ACP and sinuses could not be predicted via simple methodology.

The radiological measurements of the ACP were not the purpose of the study and therefore were knowingly not performed. Several cadaveric studies describe the length, width, and occasionally the thickness of the ACP $[8,13,15]$. Each of the diameters varied amongst the investigated populations and races. A recent study presented the measurements of the anatomical relationships of the ACP to surrounding structures [8]. The impact of ACP diameter and the distance to other structures in clinical practice is encouraging as some authors have suggested several significant landmarks to assist in proper total anterior clinoidectomies.

\section{Anterior clinoidectomy}

The investigation of our clinical series referred only to intradural anterior clinoidectomy. Both extradural and intradural methods have been described and discussed extensively in the literature. Better control while removing the ACP, avoidance of intradural drilling, safety of surrounding structures, and straightforward access to the proximal part of the ICA after dural opening are stated as advantages of an extradural approach [17, 26]. On the contrary, supporters of the intradural approach list constant visualisation of the ICA, improved control over the optic nerve and its canal, and the extent of the osteotomy being tailored to individual intraoperative requirements. The last argument for the intradural approaches seems to be the most important in terms of CSF rhinorrhoea avoi- 
dance. Neurosurgical clipping of paraclinoid or ophthalmic artery ICA aneurysm is mostly facilitated by total or near total ACP removal, regardless of the pneumatisation. However, Vajkoczy et al. [26] claimed that the potential for rhinorrhoea occurrence should favour removing the ACP. Thus, limited extent of bone removal in anterior clinoidectomies is a valid consideration. Romani et al. [19] recently proposed a partial removal, which started from the tip of the ACP and was limited to $1 / 3$ or $2 / 3$ of the entire process. However, the benefits of partial anterior clinoidectomy in terms of rhinorrhoea reduction, as well as the technique in partial ACP removal of its lateral aspect, have not been described in the literature to date. The comparison of CSF leakage rates between total and partial anterior clinoidectomies was not considered in our series due to the fact that none of our patients presented with rhinorrhoea during the postoperative period. The relationship between the extent of ACP removal and CSF leakage remains unexplored. Our results do not dispute that either total or partial clinoidectomies do not preclude CSF rhinorrhoea. Further studies comparing these 2 methods of ACP removal are awaited by neurosurgeons. Partial anterior clinoidectomies bring some benefits. In our opinion there is a decreased possibility to injure the surrounding structures, while ensuring enough room for safe manoeuvres. Incomplete removal of the ACP is preferable in our eyes, when it is feasible.

\section{REFERENCES}

1. Abuzayed B, Tanriover N, Biceroglu H, Yuksel O, Tanriover O, Albayram S, Akar Z (2010) Pneumatization degree of the anterior clinoid process: a new classification. Neurosurg Rev, 33: 367-373.

2. Chang DJ (2009) The "no-drill” technique of anterior clinoidectomy: a cranial base approach to the paraclinoid and parasellar region. Neurosurgery, 64 (3 suppl.): ONS96-ONS105.

3. Chi JH, Sughrue M, Kunwar S, Lawton MT (2006) The "yo-yo" technique to prevent cerebrospinal fluid rhinorrhea after anterior clinoidectomy for proximal internal carotid artery aneurysms. Neurosurgery, 59 (1 suppl. 1): ONS101-ONS107.

4. Citardi MJ, Gallivan RP, Batra PS, Maurer CR, Jr., Rohlfing T, Roh HJ, Lanza DC (2004) Quantitative computer-aided computed tomography analysis of sphenoid sinus anatomical relationships. Am J Rhinol, 18:173-178.

5. DeLano MC, Fun FY, Zinreich SJ (1996) Relationship of the optic nerve to the posterior paranasal sinuses: a CT anatomic study. Am J Neuroradiol, 17: 669-675.

6. Dolenc VV (1985) A combined epi- and subdural direct approach to carotid-ophthalmic artery aneurysms. J Neurosurg, 62:667-672.

7. Evans JJ, Hwang YS, Lee JH (2000) Pre- versus post-anterior clinoidectomy measurements of the optic nerve, internal carotid artery, and opticocarotid triangle: a cadaveric morphometric study. Neurosurgery, 46:1018-1021.
8. Huynh-Le P, Natori Y, Sasaki T (2004) Surgical anatomy of the anterior clinoid process. J Clin Neurosci, 11: 283-287.

9. Inoue T, Rhoton AL, Jr., Theele D, Barry ME (1990) Surgical approaches to the cavernous sinus: a microsurgical study. Neurosurgery, 26: 903-932.

10. Kapur E, Mehic A (2012) Anatomical variations and morphometric study of the optic strut and the anterior clinoid process. Bosn J Basic Med Sci, 12: 88-93.

11. Kim JH, Kim JM, Cheong JH, Bak KH, Kim CH (2009) Simple anterior petroclinoid fold resection in the treatment of low-lying internal carotid-posterior communicating artery aneurysms. Surg Neurol, 72: 142-145.

12. Krisht AF, Kadri PA (2005) Surgical clipping of complex basilar apex aneurysms: a strategy for successful outcome using the pretemporal transzygomatic transcavernous approach. Neurosurgery, 56 (2 suppl.): 261-273.

13. Lee HY, Chung IH, Choi BY, Lee KS (1997) Anterior clinoid process and optic strut in Koreans. Yonsei Med J, 38: 151-154.

14. Mikami T, Minamida Y, Koyanagi I, Baba T, Houkin K (2007) Anatomical variations in pneumatization of the anterior clinoid process. J Neurosurg, 106:170-174.

15. Nagasawa S, Kikuchi H, Kim NG, Yonekawa Y (1988) Analysis of internal carotid-posterior communicating artery aneurysms with difficulty in clipping: with special reference to radiometry. No Shinkei Geka, 16: 959-964.

16. Natori Y, Rhoton AL, Jr. (1995) Microsurgical anatomy of the superior orbital fissure. Neurosurgery, 36: 762-775.

17. Noguchi A, Balasingam V, Shiokawa $Y$, McMenomey SO, Delashaw JB, Jr. (2005) Extradural anterior clinoidectomy. Technical note. J Neurosurg, 102: 945-950.

18. Nutik SL (1988) Removal of the anterior clinoid process for exposure of the proximal intracranial carotid artery. J Neurosurg, 69: 529-534.

19. Romani R, Elsharkawy A, Laakso A, Kangasniemi M, Hernesniemi J (2012) Tailored anterior clinoidectomy through the lateral supraorbital approach: experience with 82 consecutive patients. World Neurosurg, 77: 512-517.

20. Sapci T, Derin E, Almac S, Cumali R, Saydam B, Karavus M (2004) The relationship between the sphenoid and the posterior ethmoid sinuses and the optic nerves in Turkish patients. Rhinology, 42: 30-34.

21. Sirikci A, Bayazit YA, Bayram M, Mumbuc S, Gungor K, Kanlikama M (2000) Variations of sphenoid and related structures. Eur Radiol, 10: 844-848.

22. Son HE, Park MS, Kim SM, Jung SS, Park KS, Chung SY (2010) The avoidance of microsurgical complications in the extradural anterior clinoidectomy to paraclinoid aneurysms. J Korean Neurosurg Soc, 48:199-206.

23. Szmuda T, Sloniewski P (2011) Early and long-term outcome of surgically treated giant internal carotid artery aneurysms - comparison with smaller aneurysms. Acta Neurochir, 153: 1611-1619.23.

24. Szmuda T, Sloniewski P, Dzierzanowski J, Rut M (2011) Predictors of postoperative mortality in ruptured aneurysms of internal carotid artery. Neurol Neurochir Pol, 45:543-555.

25. Yonekawa Y, Ogata N, Imhof HG, Olivecrona M, Strommer K, Kwak TE, Roth P, Groscurth P (1997) Selective extradural anterior clinoidectomy for supra- and parasellar processes. Technical note. J Neurosurg, 87: 636-642.

26. Vajkoczy $P$ (2012) Intradural versus extradural removal of the anterior clinoid process. World Neurosurg, 77: 615-616. 